\title{
Detección de barras rotas en motores de inducción utilizando SMCSA (Square Motor Current Signature Analysis)
}

\author{
David Alejandro Fernández Tavitas, Juan Pablo Nieto González \\ Corporación Mexicana de Investigación en Materiales, S.A. de C.V COMIMSA \\ david.fdz@comimsa.com,juan.nieto@comimsa.com
}

\begin{abstract}
Resumen. Los motores de inducción actualmente son la máquina más ampliamente usada en el campo industrial, por su robustez y fácil instalación. Es por eso que la detección de fallas toma un papel importante para un correcto mantenimiento y por consecuente el alargamiento de la vida útil del motor. Una de las principales técnicas existentes para la detección de fallas en motores de inducción es MCSA (motor current signature analysis). Dicha técnica basa su funcionamiento en el análisis de la corriente de un motor y observación de patrones que nos indican la existencia de una falla. En este artículo se presenta una comparación entre las técnicas MCSA y SMCSA (Square motor current signature analysis) y la ventaja que presenta la técnica para la detección de pequeñas fallas.
\end{abstract}

Palabras clave: Motores de inducción, barras rotas. SMCSA.

\section{Introducción}

Uno de los elementos más importantes dentro del ámbito industrial son los motores de inducción. Dichas máquinas usualmente trabajan bajo severas condiciones (térmicas, eléctricas, mecánicas, ambientales, etc.) Lo cual puede provocar la presencia de fallas en el estator y/o el rotor.

Estadísticas de fallas [1] reportan que los componentes del motor que tienden a fallar con más frecuencia son: Estator (38\%), Rotor (10\%), Rodamientos (40\%), Otros $(12 \%)$

La ruptura de barras en el rotor representa un $10 \%$ de las fallas de un motor, dependiendo de su severidad pueden ir desde el mal funcionamiento hasta la detención total del motor y a su vez un paro de línea causando pérdidas económicas dentro de la empresa. [2,3] Existen diversas técnicas y metodologías para la detección de fallas en el motor como la Impedancia de secuencias negativas, análisis del espectro de frecuencias, el vector de Perk [4], la transformada de Hilbert [1] análisis de la firma eléctrica [6] entre otros. Una de las técnicas más ampliamente usadas es MCSA, consiste en el análisis de la corriente del motor detectando bandas 
laterales sobre la frecuencia principal que señalan la presencia de una anomalía. Sin embargo la técnica MCSA presenta limitaciones para detectar fallas en caso de que la falla sea muy pequeña o la corriente del motor sea muy reducida. En este artículo se realiza la comparación de la técnica MCSA con SMCSA para la detección de barras rotas del rotor, presentando ventaja la segunda técnica para corrientes de reducido tamaño o para un número reducido de barras.

La experimentación fue realizada mediante la simulación de un motor trifásico de $220 \mathrm{v}, 30 \mathrm{~Hz}$ y poniendo en cortocircuito las resistencias de una fase en representación de la perdida de corriente por las barras rotas del motor.

El presente artículo está organizado de la siguiente forma: la sección 2 y 3 presentan una breve explicación de las teorías a comparar, la sección número 4 muestra los preliminares matemáticos utilizados para el pos-procesamiento de las señales obtenidas, la sección número 5 señala la fase de experimentación, en la sección número 6 los resultados obtenidos y la sección número 7 presenta las conclusiones del proyecto.

\section{MCSA (Motor Current Signature Analysis)}

Para la detección de fallas del rotor es necesario utilizar un espectro de frecuencia, el cual contiene importante información acerca del funcionamiento del motor. Al observar los armónicos obtenidos de la corriente es posible detectar anomalías o variaciones presentes en la frecuencia la cual indica un mal funcionamiento, dichos armónicos muestran diferentes espectros dependiendo de la falla del motor así como de su severidad [6,7].

\subsection{Estado de operación normal}

Considerando a un motor trifásico en condiciones ideales de voltaje y potencia podemos analizar la corriente en estado de operación normal mediante la fórmula (1)

$$
i_{a}(t)=\sqrt{2} I_{s} \sin \left(\omega_{s} t-\varphi\right)
$$

donde:

$\varphi$ Es el ángulo de fase entre el voltaje y la línea de corriente,

$f_{s}$ Es la frecuencia fundamental,

$\omega_{s}$ Es la pulsación fundamental $\omega_{s}=2 \pi f_{s}$.

El espectro de la corriente de la fase solo presentará el componente principal de la frecuencia.

\subsection{Estado de falla}

Manteniendo las mismas condiciones del motor es posible detectar la falla examinando los componentes laterales en el armónico donde son resaltados componentes en las frecuencias $(1+2 s) f_{s}$ como se muestra en la Fig. 1. La corriente del motor es expresada por: 


$$
i_{a}(t)=I_{\max } \cos (\omega t)+I_{l s b} \cos [(1-2 s) \omega t]+I_{u s b} \cos [(1+2 s) \omega t]
$$

$I_{\max } \quad$ Es el valor máximo de la corriente fundamental.

$I_{l s b} \quad$ Es el valor máximo del componente fundamental más pequeño.

$I_{u s b} \quad$ Es el valor máximo del componente fundamental más grande.

$s \quad$ Es el deslizamiento.

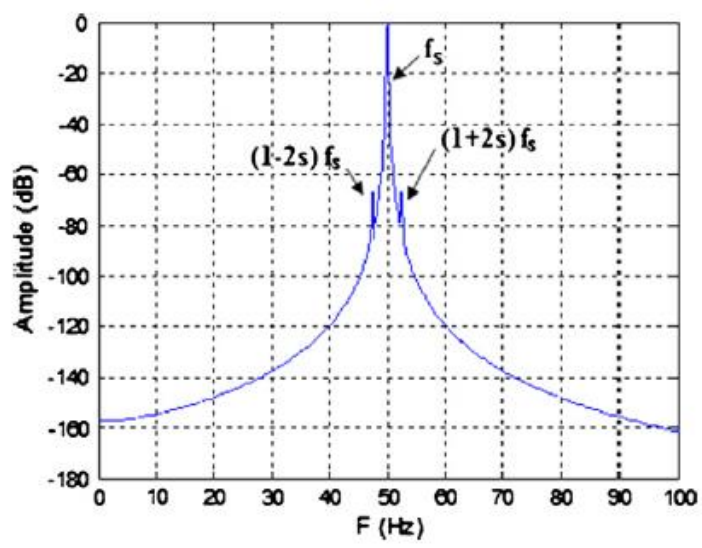

Fig. 1. Espectro de frecuencia barras rotas del rotor (adaptada de [8]).

\section{SMCSA (Square Motor Current Signature Analysis)}

Tal como en la técnica MCSA se realiza un análisis del espectro de frecuencias sobre la corriente del estator, sin embargo la técnica presentada utiliza el cuadrado de la corriente, lo cual facilita la detección de patrones indicadores de fallas existentes [8].

La técnica basa su metodología en 3 pasos principales. 1) La adquisición de la corriente de fase del estator en funcionamiento, 2) El procesamiento del cuadrado de la corriente obtenida mediante las fórmulas (3) y (4) y 3) El análisis del cuadrado de la corriente mediante espectros de frecuencia con el uso de la transformada de Fourier (sección 4.1).

\subsection{Estado de operación normal}

Mediante la fórmula (3) es posible hacer un procesamiento de la corriente de fase para conocer su comportamiento en su modo de operación normal.

$$
i_{a}^{2}(t)=\frac{I^{2} \max }{2}+\frac{I^{2}{ }_{\max } \cos (\omega t)}{2}
$$


donde:

$I_{\max }$ Es el valor máximo de la corriente fundamental,

$\omega \quad$ Es la pulsación fundamental $\omega_{s}=2 \pi f_{s}$.

Mediante la técnica SMCSA es posible detectar en el armónico una única corriente fundamental en la frecuencia $2 f_{s}$ adjunta a un componente DC como se presenta en la Fig. 2.

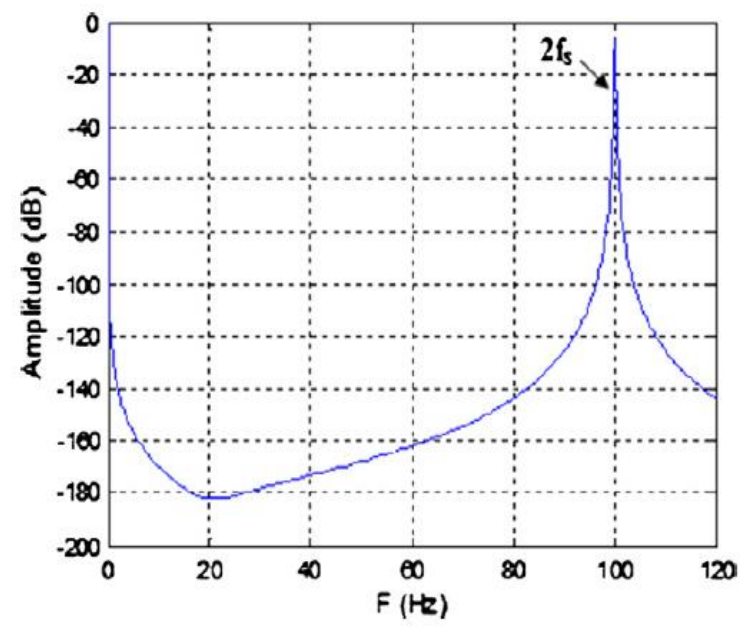

Fig. 2. Espectro de frecuencia modo de operación normal (adaptada de [8]).

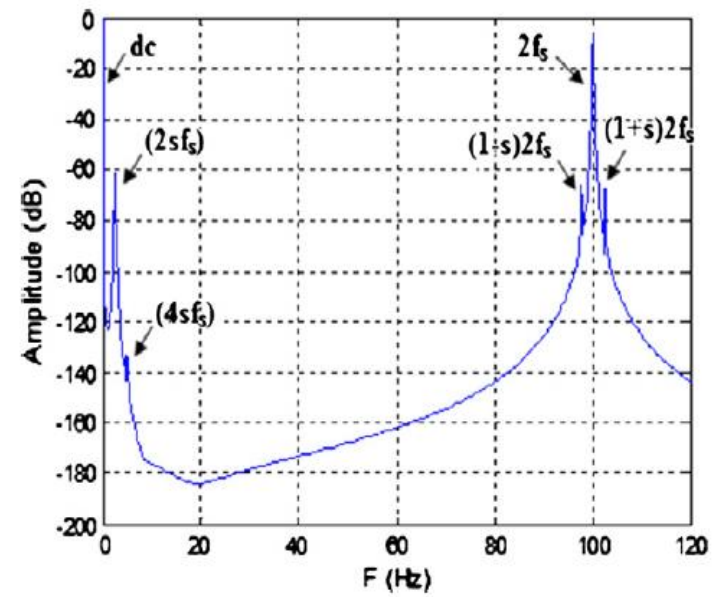

Fig. 3. Espectro de frecuencia modo de operación normal (adaptada de [8]). 


\subsection{Estado de falla}

Bajo las mismas condiciones un motor de inducción que presenta falla en el rotor, específicamente con barras rotas presentes permite determinar la corriente mediante la fórmula (4), [8].

$$
\begin{aligned}
& i_{a}^{2}(t)=\left(\frac{I^{2}{ }_{\text {max }}}{2}+\frac{I^{2}{ }_{l s b}}{2}+\frac{I^{2}{ }_{u s b}}{2}\right)+\left(\frac{I_{\text {max }}^{2}}{2}+I_{l s b} I_{u s b}\right) \cos (2 \omega t) \\
& +\left(I_{\text {max }} I_{l s b}+I_{\text {max }} I_{u s b}\right) \cos (\omega t)+I_{l s b} I_{u s b} \cos (4 s \omega t)+I_{\text {max }} I_{l s b} \cos (2(1-s) \omega t) \\
& +I_{\text {max }} I_{u s b} \cos (2(1+s) \omega t)+\frac{I^{2}{ }_{l s b}}{2} \cos (2(1-2 s) \omega t)+\frac{I^{2} u s b}{2} \cos (2(1+2 s) \omega t)
\end{aligned}
$$

En la Fig. 3 se muestra un componente DC, un componente en la frecuencia $2 f_{s}$, componentes laterales en las $2(1 \pm 2 s) f_{s}$ y adicionalmente se muestran componentes laterales en las frecuencias $2 s f_{s}$ y $4 s f_{s}$ dependiendo de la severidad de la falla presente.

\section{Preliminares matemáticos}

\subsection{Transformada de Fourier}

La transformada de Fourier es una herramienta matemática utilizada para convertir una señal en el dominio del tiempo a una señal en el dominio de frecuencia con el fin de observar el comportamiento de una función en un tiempo específico [10]. Es denotada $h(t)$ Como una señal continua en el tiempo la cual se encuentra entre 2 señales consecutivas en $T$ segundos, es posible obtener su transformada mediante la fórmula (5)

$$
H(\omega)=\sum_{n=-\infty}^{\infty} h(n T) e^{-j \omega n}
$$

donde

$\omega$ es la frecuencia normalizada en radianes $(\omega=\Omega T)$,

$\Omega$ es la frecuencia en radianes/segundos $(\Omega=2 \pi f)$,

$T$ es un periodo de tiempo.

\subsection{Transformada rápida de Fourier}

La transformada rápida de Fourier es un algoritmo que permite evaluar los componentes armónicos de una señal discreta periódica, puede ser evaluada mediante la fórmula (6) 


$$
H(k)=\sum_{n=0}^{N-1} h(n) e^{\frac{j 2 \pi}{N} n k}
$$

donde

$N$ es el número de coeficientes,

$k$ es un integrador $k=0, \ldots, N-1$.

\subsection{Velocidad de deslizamiento}

El voltaje inducido en una barra del rotor de un motor de inducción depende de la velocidad del rotor con respecto a los campos magnéticos .El comportamiento de un motor de inducción depende del voltaje y la corriente del rotor, conocido como la velocidad relativa. [11] menciona que el deslizamiento se define como la velocidad relativa expresada sobre una base por unidad o porcentaje, el deslizamiento se expresa mediante la fórmula (7)

$$
s=\frac{n_{\operatorname{sinc}}-n_{m}}{n_{\operatorname{sinc}}}(\times 100 \%)
$$

donde $s$ es el deslizamiento,

$n_{\text {sinc }}$ velocidad de los campos magnéticos,

$n_{m}$ velocidad del eje del motor.

\section{Fase de experimentación}

Para la experimentación fue realizada la simulación de un motor trifásico de inducción de $220 \mathrm{~V}, 2.5 \mathrm{~A}$, dos polos, y una frecuencia de $30 \mathrm{~Hz}$, conexión tipo estrella. En una de las fases del motor se establecieron una serie de cortocircuitos entre las resistencias de dicha fase las cuales fueron utilizadas para simular barras rotas en el rotor mediante el cambio de fluctuación de la corriente, además se definieron diferentes puntos de conexión para cada una de las resistencias, denotando así el grado de severidad en las fallas, Fig. 4 [10].

Tabla 1. Puntos de conexion y severidad de fallas.

\begin{tabular}{|c|c|c|}
\hline Severidad & Porcentaje de corto & Puntos de conexión \\
\hline \hline Sin falla & $0 \%$ & $1-2,3-4,5-6$ \\
\hline \hline Severidad baja & $5 \%$ & $3-4-5-6,7-8$ \\
\hline \hline Severidad alta & $10 \%$ & $3-4-7-8,5-6$ \\
\hline
\end{tabular}

Se hicieron pruebas en estado de operación normal y estado de falla para diversos niveles de severidad aplicando ambas técnicas de detección.

Los valores adquiridos del motor fueron pre-procesados mediante el análisis de corriente con el uso de la técnica MCSA y analizadas en base a la frecuencia mediante 
el uso de la transformada rápida de Fourier (FFT), en donde se resaltan la frecuencia principal y sus componentes laterales (definidos como aumentos o decrementos de amplitud en la corriente presentes en los costados de frecuencia principal), para cada uno de los grados de operación. Posteriormente se realizó el mismo procedimiento para la técnica SMCSA poniendo en comparación los resultados obtenidos por ambas metodologías.

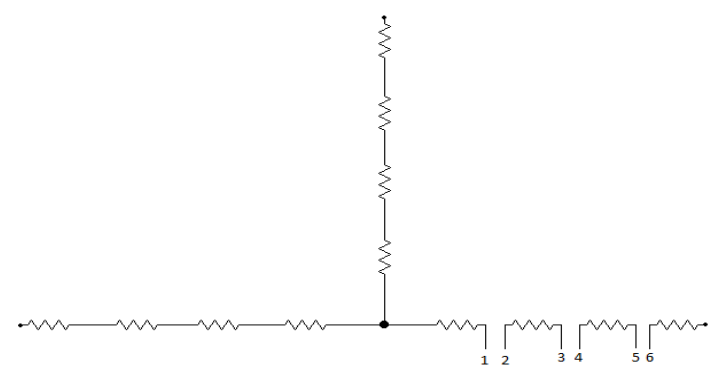

Fig. 4. Conexión estrella con cortocircuitos presentes en una de las fases.

\section{Resultados del experimento}

Con el propósito de demostrar la eficiencia de una técnica sobre otra se realizaron diversas pruebas para cada una de las metodologías mencionadas obteniendo los siguientes resultados.

En la Fig. 5 se muestran los espectros de frecuencia en estado de operación normal para ambas técnicas, en donde es posible detectar un aumento de frecuencia en el valor $f_{s}$ para la técnica MCSA, y un aumento de frecuencia en $2 f_{s}$ así como un componente DC para la técnica SMCSA.

En la figura (6) fue realizado el mismo procedimiento, análisis de ambas técnicas para un grado de severidad alto (10\%) presentando en ambas técnicas buenos resultados, siendo favorecida la técnica SMCSA en referencia a la resolución del análisis. En la figura (7) se puede observar un aumento de amplitud en $f_{s} \mathrm{y}$ componentes laterales en las frecuencias $(1-2 s) f_{s}$ y $(1+2 s) f_{s}$ para la técnica MCSA, indicando la presencia de una falla en el motor, específicamente barras rotas en el rotor. En el caso de la técnica SMCSA es posible detectar un aumento de amplitud en el cuadrado de la corriente equivalente a $2 f_{s}$ y componentes laterales en las frecuencias $(1 \pm 2 s), 2 f_{s}(1 \pm 4 s), 2 f_{s}(1 \pm 6 s) f_{s}$.

La ventaja que muestra la técnica SMCSA sobre MCSA se presenta al momento de trabajar con niveles de severidad bajos en donde el análisis por MCSA no es capaz de resaltar señales que indiquen la presencia de una falla mientras que el cuadrado de dicha técnica muestra componentes relevantes para detección de la falla. En la figura (7) se presenta el análisis de frecuencias con un grado de severidad al $5 \%$ y un nivel de corriente bajo denotando aumentos de amplitud en los valores $2 f_{s}, 4 f_{s}, 6 f_{s}$ y $8 f_{s}$ para la técnica SMCSA los cuales nos indican la existencia de barras rotas en el rotor. 
David Alejandro Fernández Tavitas, Juan Pablo Nieto González
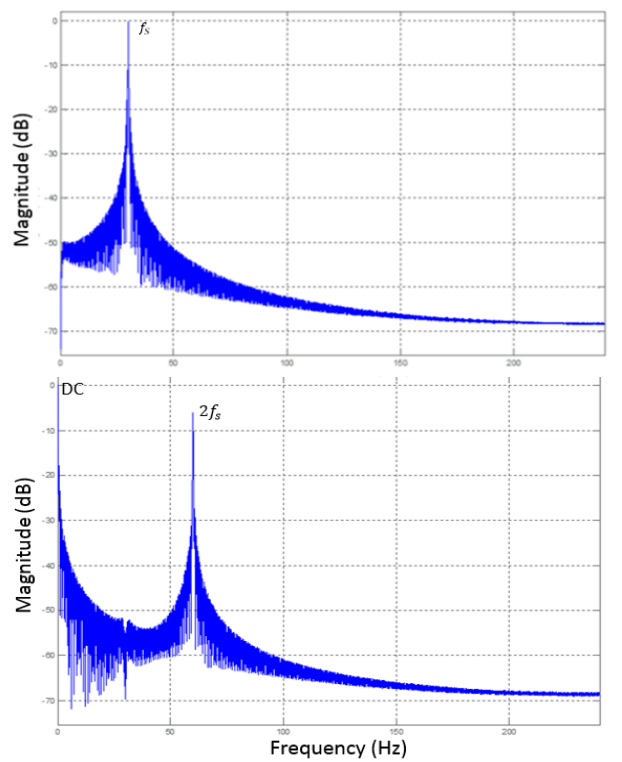

Fig. 5. Espectro de frecuencia estado de operación normal a) MCSA b) SMCSA.
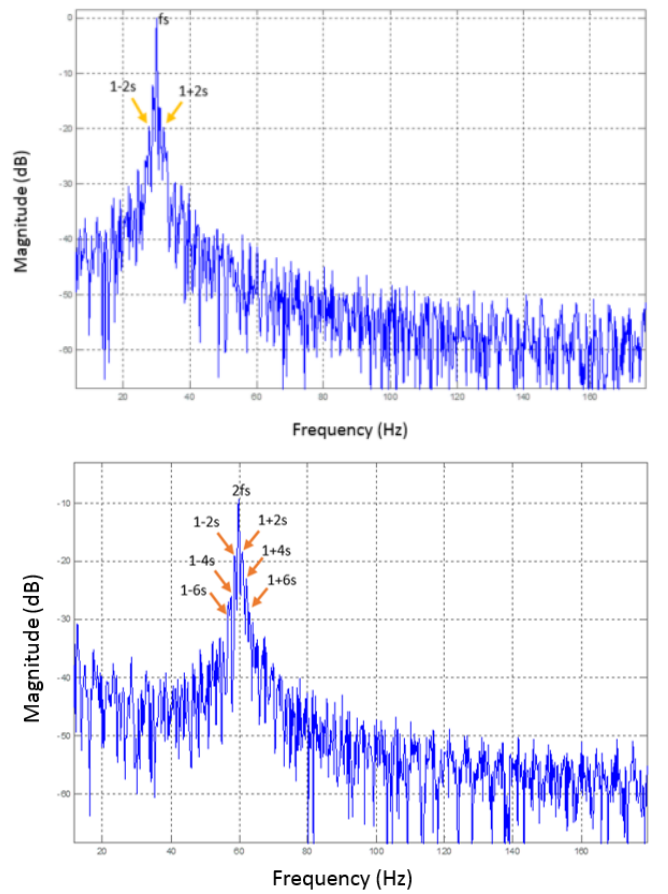

Fig. 6. Espectro de frecuencia 10\% de severidad a) MCSA b) SMCSA. 

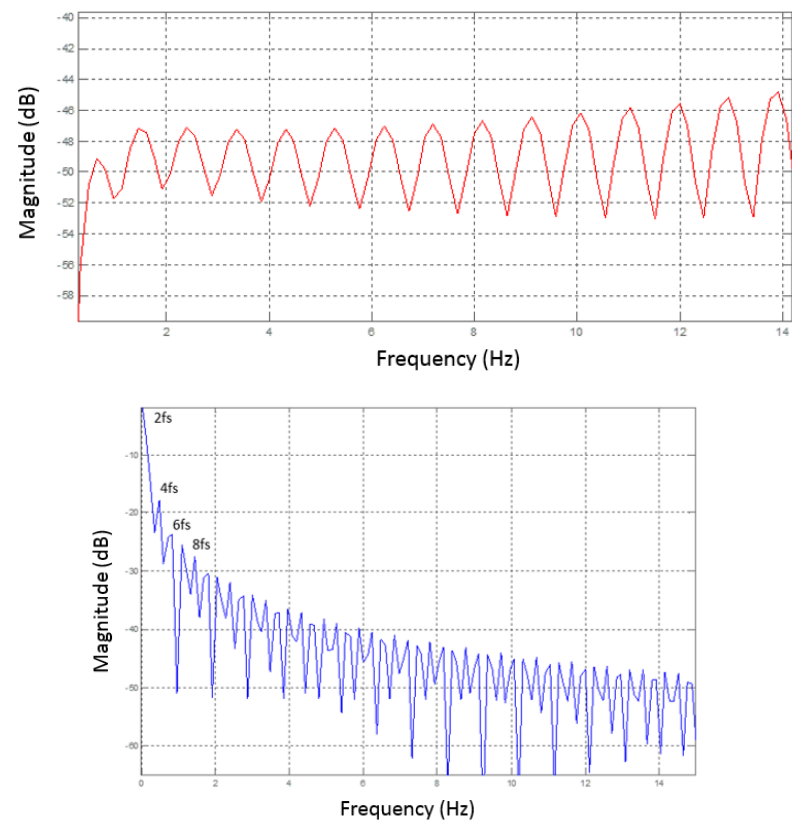

Fig. 7. Espectro de frecuencia $5 \%$ de severidad a) MCSA b) SMCSA.

\section{Conclusiones}

En este artículo se presenta una comparación entre las técnicas MCSA y SMCSA para el análisis de fallas en motores eléctricos, específicamente detección de barras rotas en el rotor. Las técnicas expuestas basan su funcionamiento en 3 pasos principales, la adquisición de la corriente de fase del motor, el procesamiento de la corriente para la obtención de los componentes principales y finalmente el análisis de las corrientes obtenidas del procesamiento de ellas.

Fueron presentadas diversos modos de operación, desde modo de operación normal hasta modo de falla en diversos niveles de severidad, demostrando resultados notables en ventaja de la técnica SMCSA para fallas con altos niveles de severidad, en los cuales los patrones que nos indican la existencia de una falla se presentan en mayor cantidad, demostrando una mejor resolución al momento del análisis, y principalmente para fallas con bajos niveles de severidad o de condiciones de corriente reducida, en los cuales la técnica MCSA no es capaz de señalar la presencia de alguna falla, en caso contrario, la técnica SMCSA presenta patrones en frecuencias específicas, las cuales indican la existencia de una falla.

\section{Referencias}

1. R. Puche-Panadero, M. Pineda-Sanchez, M. Rierra-Guasp, J. Roger-Folch, E. HurtadoPérez and J. Pérez-Cruz: Improved Resolution of the MCSA Method Via Hilbert 
David Alejandro Fernández Tavitas, Juan Pablo Nieto González

Transform, Enabling the Diagnosis of Rotor Asymmetries at Very Low Slip. IEEE Transactions on Energy Conversion, (2009) pp. 52-59.

2. S. Guedidi, S.E.Zouzou, W. Laala, M. Sahraoui, K. Yahia: Broken Bar Fault Diagnosis of Induction Motors Using MCSA and Neural Network. In: 2011 IEEE International Symposium on Diagnostics for Electric Machines, Power Electronics \& Drives (SDEMPED), (2011) pp. 632-637.

3. Jose Rangel-Magdaleno, Juan Ramirez-Cortes and Hayde Peregrina-Barreto: Broken Bars Detection of Induction Motor Using MCSA and Mathematical Morphology: An Experimental Study. In: 2013 IEEE International Instrumentation and Measurment Technology Conference (I2MTC), (2013) pp. 825-829.

4. C. J. Verucchi and G. G. Acosta: Técnicas de Detección y Diagnóstico de Fallos en Máquinas Eléctricas de Inducción. En: IEEE Latin American Transactions (2007).

5. Prabhakar Neti, Manoj R. Shah, Karim Younsi, John Krahn, Joe Yingneng Zhou and C. David Whitefield: Motor Current Signature Analysis During Accelerated Life Testing of Form Wound Induction Motors. In: 2010 IEEE International Power Modulator and High Voltage Conference (IPMHVC), (2010) pp. 106-109.

6. Prabhakar Neti, Pinjia Zhang, Manoj Shah and Karim Younsi: Electrical Signature Analysis Based Online Monitoring of Drive-trains for Doubly-fed Wind Generators. In: IECON 2012 - 38th Annual Conference on IEEE Industrial Electronics Society, (2012) pp. $1764-1769$.

7. G. Didier, E. Ternisien, O. Caspary and H. Razik: A new Approach to detect broken rotor bars in induction machnes by current spectrum analysis. Mechanical Systems and Signal Processing, Elsevier, (2007) pp. 1127-1142.

8. R.Saravana Kumar, Dr K.K.Ray and K.Vinoth Kumar: Fault Diagnosis of Industrial Drives Using MCSA Techniques. In: 2009 International Conference on Control, Automation, Communication and Energy Conservation, INCACEC, (2009) pp. 1-7.

9. V. Fernão Pires, Manuel Kadivonga, J.F. Martins and A.J. Pires: Motor square current signature analysis for induction motor rotor diagnosis. Measurment, (2013) pp. 942-948.

10. Reddy, V. U.: On Fast Fourier Transform, A popular tool for spectrum analysis. General Article (1998).

11. Stephen J. Chapman: Máquinas Eléctricas. Australia: Mc. Graw Hill, Tercera Edición, (2000).

12. Jee-Hoon Jung, Lee Jong-Jae and Bong-Hwan Kwon: Online Diagnosis of Induction Motors Using MCSA. IEEE Transactions on Industrial Electronics, Vol. 53, (2006) pp. $1842-1852$.

13. M. Sahraoui, S.E. Zouzou, A. Ghoggal, S. Guedidi and H. Derghal: An improved Algorithm for Detection of Rotor Faults in Squirrel Cage Induction Motors Based on a New Fault Indicator. In: 2012 XXth International Conference ON Electrical Machines (ICEM), (2012) pp. 1572-1578. 